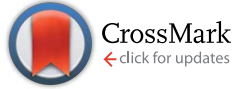

Cite this: Soft Matter, 2015, 11, 3279

\title{
Electrostatic swelling of bicontinuous cubic lipid phases $\dagger$
}

\author{
Arwen I. I. Tyler, Hanna M. G. Barriga, Edward S. Parsons, Nicola L. C. McCarthy,
} Oscar Ces, Robert V. Law, John M. Seddon* and Nicholas J. Brooks*

Lipid bicontinuous cubic phases have attracted enormous interest as bio-compatible scaffolds for use in a wide range of applications including membrane protein crystallisation, drug delivery and biosensing. One of the major bottlenecks that has hindered exploitation of these structures is an inability to create targeted highly swollen bicontinuous cubic structures with large and tunable pore sizes. In contrast, cubic structures found in vivo have periodicities approaching the micron scale. We have been able to engineer and control highly swollen bicontinuous cubic phases of spacegroup $1 \mathrm{~m} 3 \mathrm{~m}$ containing only lipids by (a) increasing the bilayer stiffness by adding cholesterol and (b) inducing electrostatic repulsion across the water channels by addition of anionic lipids to monoolein. By controlling the composition of the ternary mixtures we have been able to achieve lattice parameters up to $470 \AA$, which is 5 times that observed in pure monoolein and nearly twice the size of any lipidic cubic phase reported previously. These lattice parameters significantly exceed the predicted maximum swelling for bicontinuous cubic lipid structures, which suggest that thermal fluctuations should destroy such phases for lattice parameters larger than $300 \AA$.

Received 5th February 2015

Accepted 10th March 2015

DOI: $10.1039 / \mathrm{c} 5 \mathrm{sm} 00311 \mathrm{c}$

www.rsc.org/softmatter
Highly curved membranes exist in organelles such as the Golgi network and mitochondria ${ }^{2}$ and cubic membranes have been observed in a number of cell types (ref. 1 and references therein). Non-lamellar phases are of considerable biological relevance as their formation is essential for a large number of important biological processes to occur such as exocytosis, endocytosis, viral fusion, cell division and protein function, to name but a few. ${ }^{1,3}$ Cubic phases have been observed as an intermediate during fat digestion, ${ }^{4}$ in lipid extracts from A. Laidlawii and E. coli, ${ }^{5,6}$ and micron sized cubic phases have been observed in the inner mitochondrial membranes of starved amoeba ${ }^{7}$ as well as the organized smooth endoplasmic reticulum. ${ }^{1}$ The colour of certain butterflies is due to photonic crystals within the wing cells that have been generated by templating of chitin gyroid cubic networks within the endoplasmic reticulum. ${ }^{8}$
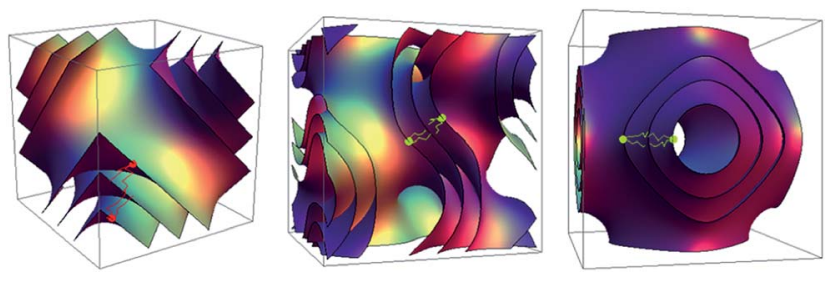

Fig. 1 The minimal surfaces that the three lipid inverse bicontinuous cubic phases are based on. From left to right: diamond $(P n 3 m)$, gyroid $(/ a 3 d)$ and primitive $(/ \mathrm{m} 3 \mathrm{~m})$. 
Bicontinuous cubic phases have attracted a great deal of biotechnical interest due to their very high surface area, highly interconnected and accessible pore network and soft selfassembled structure that can undergo structural rearrangement. Due to the amphiphilic nature of the lipids that make up bicontinuous cubic phases, hydrophilic, hydrophobic and amphiphilic bioactive species can all be encapsulated in them $^{9-11}$ and as a result they have been used as matrices for templating, ${ }^{12}$ biosensing ${ }^{13}$ and for membrane protein crystallization. $^{\mathbf{1 4 , 1 5}}$

One of the major bottlenecks hindering further exploitation of these structures is the inability to create highly swollen bicontinuous cubic structures with large and tuneable pore sizes. ${ }^{16}$ Monoacylglycerides, and particularly monoolein (MO), are some of the most common lipids used to generate cubic structures as they form at least 2 out of the 3 bicontinuous cubic phases depending on the water content and temperature. ${ }^{\mathbf{1 7 , 1 8}}$ These structures typically have aqueous channel diameters around $40 \AA$, which prevents integration of cargo larger than this. This is a significant problem in many applications such as crystallization of membrane proteins with large extramembrane domains, ${ }^{\mathbf{1 9}, 20}$ encapsulation of many enzymes and selective separation of large molecules. In order to exploit these structures to their full potential it is vital to be able to engineer highly swollen cubic phases with length scales approaching those in vivo; cubic structures found in nature can be over 100 times larger than current in vitro, pure lipid structures. Considerable efforts have been made towards swelling bicontinuous cubic phases. Examples include the addition of hydration modulating agents such as octyl glucoside, ${ }^{21}$ sucrose stearate, ${ }^{22,23}$ neutral lipids $^{24}$ and cholesterol (CHOL) ${ }^{24}$ as well as charged lipids, ${ }^{11,24-27}$ charged lipid-like peptides ${ }^{\mathbf{2 8}}$ and nucleic acids. ${ }^{\mathbf{1 1}}$ Despite this, it has not previously been possible to produce an equilibrium single-phase lipid bicontinuous cubic structure with a lattice parameter exceeding $270 \AA^{25}$

Until recently, swollen lipidic bicontinuous cubic phases with lattice parameters of 300 to $400 \AA$ have only ever been observed as transient structures or in coexistence with other phases. ${ }^{29,30}$ We have recently formed examples of highly swollen bicontinuous cubic phases at equilibrium in hydrated lipid mixtures incorporating charged phospholipids and cholesterol. ${ }^{31}$ We have now been able to both control the structural properties of these systems by changing their lipid composition and explore the physicochemical properties and interactions that underpin this unprecedented structural swelling.

Depending on the membrane rigidity, bilayer thickness and temperature, calculations have suggested that thermal fluctuations will destroy cubic phases with lattice parameters larger than approximately 250-300 $\AA^{32}$ More recent work suggests that larger cubic structures may be stable in neutral lipid systems in excess water, however these are yet to be found experimentally. ${ }^{33}$ It should be noted that the very large cubic phases found in nature are thought to be stabilized by protein scaffolds $\mathbf{s}^{\mathbf{1 , 2}}$ and while bicontinuous cubic phases with lattice parameters on the order of $500 \AA$ have been observed using block copolymers, ${ }^{34}$ these are not biomimetic and hence less relevant for biological applications.
Repulsive electrostatic interactions are known to swell lamellar phases, with extent of this effect being dependent on the Debye length of the solvent and the bilayer charge density ${ }^{35}$ however much less is known about swelling of 3-D bicontinuous cubic phases. It is known that the stability of the inverse bicontinuous cubic phases is governed by the thickness, curvature and rigidity of the membrane and in principle, inverse bicontinuous phases with sufficiently stiff, yet fluid bilayers should be able to swell indefinitely in water without incurring a large chain packing cost. Thermal fluctuations tend to destroy translational ordering in bicontinuous cubic phases with large lattice parameters, ${ }^{32}$ but incorporation of cholesterol (CHOL) will tend to stiffen the fluid membrane and so reduce this effect. In addition, incorporation of small amounts of charged phospholipids will both reduce thermal fluctuations and swell the structure due to long-range electrostatic repulsions.

In this work, we have been able to engineer inverse bicontinuous cubic phases with lattice parameters of almost $500 \AA$ in monoolein based systems by incorporating both CHOL and charged phospholipid (either dioleoylphosphatidylglycerol (DOPG) or dioleoylphosphatidylserine (DOPS)).

In addition to stiffening and swelling the membrane, CHOL, DOPG and DOPS are likely to be highly important in future application of highly swollen bicontinuous cubic phases. While MO is the archetypal bicontinuous cubic phase forming lipid, it is not common in bio-membranes and it has been found previously that doping with other lipid components is necessary for successful in-meso protein crystallization and enzyme incorporation; ${ }^{24}$ for example, doping MO with CHOL was crucial in obtaining structure-grade crystals of several G protein coupled receptors (GPCRs) (ref. 15 and references therein). The plasma membrane consists of approximately $50 \% \mathrm{CHOL}^{36}$ and many proteins are thought to require CHOL for stability, function and recruitment. ${ }^{37,38}$ Moreover, many enzymes require phosphatidylserine or phosphatidylglycerol for activation. ${ }^{39}$

By engineering and controlling highly swollen bicontinuous cubic phases we hope to pave the way for these structures to be used for containment and crystallization of large membrane proteins as well as more far reaching applications such as biocontainment and delivery, and tuneable photonic devices.

\section{Results and discussion}

\section{Effect of cholesterol on the phase behavior of monoolein}

The phase behaviour of MO has been extensively studied previously; ${ }^{17,18}$ when hydrated with more than 35 wt\% water, it forms a $P n 3 m$ cubic phase between approximately 20 and $80{ }^{\circ} \mathrm{C}$. Above this temperature it transforms to an inverse hexagonal $\left(\mathrm{H}_{\mathrm{II}}\right)$ phase. CHOL in excess water is known to exist as crystals of cholesterol monohydrate up to $86{ }^{\circ} \mathrm{C}$ which then transform to the anhydrous form above this temperature. ${ }^{40}$

There has also been work on the effect of CHOL on the phase behaviour of $\mathrm{MO}^{24}$ in the context of developing protein crystallization matrices; however this has been limited to experiments at 4 and $20^{\circ} \mathrm{C}$. At $20^{\circ} \mathrm{C}$ and $60 \mathrm{wt} \%$ water, MO : CHOL mixtures have been seen to adopt a $\mathrm{Pn} 3 \mathrm{~m}$ phase at CHOL concentrations 
up to $23 \mathrm{~mol} \%$ of the total lipid component, between 23 and $28 \mathrm{~mol} \% \mathrm{CHOL}$, an $\operatorname{Im} 3 \mathrm{~m}$ phase coexists with the Pn3m phase and above $28 \mathrm{~mol} \%$ CHOL an Im3m phase coexists with excess CHOL crystals. ${ }^{24}$ Additionally, the hydrogen bonding interactions between $\mathrm{MO}$ and $\mathrm{CHOL}$ have been examined recently ${ }^{\mathbf{4 1}}$ and it has been shown that the self-diffusion coefficient of MO in a cubic phase is reduced upon addition of CHOL. ${ }^{42}$

While these results give a great deal of useful information on the interactions of $\mathrm{MO}$ and $\mathrm{CHOL}$, elucidating the structural behaviour of these systems over a wider range of conditions (particularly at higher temperatures) is essential both for exploring their structural landscape and developing their use in bio-technical applications.

Fig. 2 shows the variation in the structure and lattice parameter of MO with increasing amounts of CHOL at $70 \mathrm{wt} \%$ water and $35{ }^{\circ} \mathrm{C}$. At $35{ }^{\circ} \mathrm{C}$ and below $15 \mathrm{~mol} \% \mathrm{CHOL}$, the MO : CHOL mixture adopts a Pn3m phase, at $15 \mathrm{~mol} \% \mathrm{CHOL}$ and above, the $P n 3 m$ phase coexists with an $\operatorname{Im} 3 m$ phase (no excess CHOL crystals were observed up to $25 \mathrm{~mol} \%$ ). The lattice parameter of the $P n 3 m$ phase increases from $96.7 \AA$ in pure MO to a value of $133.2 \AA$ at $25 \mathrm{~mol} \% \mathrm{CHOL}$ and the lattice parameter of the $\operatorname{Im} 3 \mathrm{~m}$ phase also increases with increasing CHOL concentration from $152.0 \AA$ at $15 \mathrm{~mol} \%$ (where it is first observed) to $171.1 \AA$ at $25 \mathrm{~mol} \% \mathrm{CHOL}$.

It has been shown that in $\mathrm{MO}: \mathrm{CHOL}$ mixtures hydrated with $40 \mathrm{wt} \%$ water, the hydroxyl group of $\mathrm{CHOL}$ preferentially forms hydrogen bonds with the carbonyl group of MO's glycerol headgroup instead of bulk water (as it does when mixed with phospholipids). ${ }^{41}$ This is likely to cause the bulky sterol backbone to interact strongly with the lipid hydrocarbon chains, inhibiting trans-gauche isomerization along a large proportion of the chain and so reducing the lateral pressure in the hydrophobic region of the bilayer. This will cause a reduction in the magnitude of the spontaneous monolayer curvature, promoting larger lattice parameters. In addition, CHOL will force MO's glycerol headgroups apart, reducing hydrogen bonding interactions between adjacent MO molecules which may result in

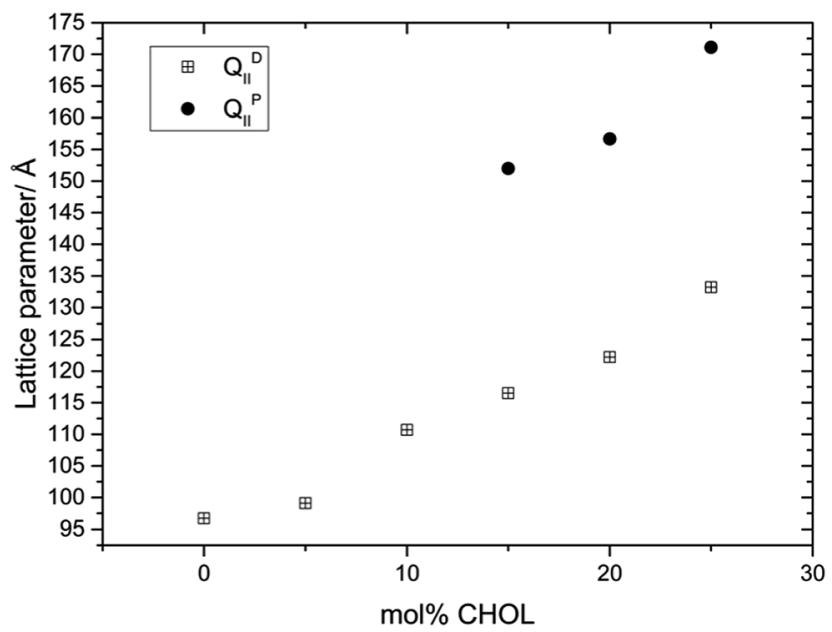

Fig. 2 Phase behavior and effect on the lattice parameter of incorporating $\mathrm{CHOL}$ into $\mathrm{MO}$. increased hydration of the MO headgroups which would again give rise to swelling of the structure.

This can be summarized by eqn (1) and (2) below which relate the surface-average Gaussian curvature, $\langle K\rangle$, to the structural parameters of the lipids..$^{22,43}$

$$
\begin{gathered}
\langle K\rangle=\frac{3}{2 l^{2}}(1-P) \\
P=\frac{v}{a_{\mathrm{o}} l}
\end{gathered}
$$

where $P$ is the lipid packing parameter, $v$ is the molecular volume of the fluid hydrocarbon chain(s), $l$ is the length of a fully extended hydrocarbon chain and $a_{\mathrm{o}}$ is the interfacial area per molecule at the polar-non-polar interface.

The Infinite Periodic Minimal Surfaces (IPMS) that the bicontinuous cubic phases are based on are characterized by a zero mean curvature $(H)$ over the surface but a varying Gaussian curvature $(K)$ which ranges from zero at flat points and becomes more negative moving towards the saddle points. Hence, increasing the interfacial area per molecule at the polar-nonpolar interface by the addition of CHOL decreases the packing parameter and increases $\langle K\rangle$ (i.e. makes it less negative). Consequently this decreases the spontaneous inverse curvature (towards water). This will tend to drive a transition from the $P n 3 m$ phase observed in pure MO to the $\operatorname{Im} 3 m$ phase which has larger radii of curvature when modelled as a constant mean curvature surface (see ESI $\dagger$ for further information). ${ }^{43}$ In addition, it is known that increasing headgroup hydration drives the transition from the $I a 3 d$ to $P n 3 m$ and then $\operatorname{Im} 3 m$ phases due to changes in the Gaussian curvature. ${ }^{44}$ Consequently, the increased MO headgroup hydration caused by CHOL interactions described above rationalizes the phase sequence observed by increasing the amount of CHOL in the structure.

It has been shown ${ }^{45}$ that by using the volume fraction of lipid, $\varphi_{\mathrm{L}}$, and the lattice parameter, $a$, one can calculate the lipid length, $l$ (eqn (3)):

$$
\varphi_{\mathrm{L}}=2 A_{0}\left(\frac{l}{a}\right)+\frac{4 \pi \chi}{3}\left(\frac{l}{a}\right)^{3}
$$

where $A_{0}$ is the ratio of the area of the minimal surface in the unit cell to the quantity (unit cell volume) ${ }^{2 / 3}$ and $\chi$ is the EulerPoincare characteristic $\left(A_{0}^{P n 3 m}=1.919\right.$ and $\chi^{P n 3 m}=-2, A_{0}^{I m 3 m}=$ 2.3451 and $\left.\chi^{\operatorname{Im} 3 m}=-4\right)$.

Using calculations by Anderson et al. ${ }^{\mathbf{4 6}}$ Briggs et al. ${ }^{18}$ have derived equations to relate the water channel radius, $r_{\mathrm{w}}$, to the lattice parameter, $a$. It should be noted however that these are only estimates as they assume that the centre of the water channel runs parallel to the minimal surface. The estimated $r_{\mathrm{w}}$ equations for the Pn3m and Im3m phases are given below:

$$
\begin{aligned}
& r_{\mathrm{w}}^{P n 3 m}=0.391 a-l \\
& r_{\mathrm{w}}^{I m 3 m}=0.305 a-l
\end{aligned}
$$

The monolayer thickness of $\mathrm{MO}$ in the $\mathrm{Pn} 3 \mathrm{~m}$ phase in excess water has been calculated to be between 17 and $19 \AA$ at $25^{\circ} \mathrm{C}^{47}$ Using a value of $18 \AA$, we have estimated the water channel 
Table 1 Lattice parameters (a), water channel diameters $(D)$ and calculated water volume $(\varphi)$ for the $P n 3 m$ (subscript D) and $I m 3 m$ (subscript $\mathrm{P}$ ) phases adopted by $\mathrm{MO}: \mathrm{CHOL}$ mixtures

\begin{tabular}{lrlllll}
\hline mol\% & & & & & & \\
CHOL & $a_{\mathrm{D}} / \AA$ & $a_{\mathrm{P}} / \AA$ & $\varphi_{\mathrm{D}} \%$ & $\varphi_{\mathrm{P}} \%$ & $D_{\mathrm{w}_{\mathrm{D}}} / \AA$ & $D_{\mathrm{w}_{\mathrm{P}}} / \AA$ \\
\hline 0 & 96.7 & & 35 & & 39.6 & \\
5 & 99.1 & & 36 & & 41.5 & \\
10 & 110.7 & & 43 & & 50.6 & \\
15 & 116.5 & 152 & 44 & 48 & 55.1 & 56.7 \\
20 & 122.2 & 156.7 & 46 & 49 & 59.6 & 59.6 \\
25 & 133.2 & 171.1 & 50 & 53 & 68.2 & 68.4
\end{tabular}

diameters of the phases adopted in the MO: CHOL system (Table 1), as was done previously for a $\mathrm{MO}$ : sucrose stearate mixture. ${ }^{22}$ As with the lattice parameter, the water channel diameter increases upon CHOL addition and at $25 \mathrm{~mol} \% \mathrm{CHOL}$ is almost double that of pure MO.

The amount of water incorporated into the bicontinuous cubic structure can be calculated from the experimentally determined lattice parameters (see ESI $\dagger$ for further details). These are listed in Table 1 and comparing these values to the amount of water added during sample preparation (70 wt\%) indicates all the MO : CHOL sample coexist with excess water and that their structural parameters are not limited by the available solvent.

It should be noted that the molecular parameters required for these calculations are not known for MO : CHOL mixtures, so we have used those for pure MO.

\section{Effect of DOPS and DOPG incorporation on the phase behaviour of monoolein}

Although electrostatic and fluctuation interactions are known to swell lamellar phases, swelling of 3-D bicontinuous cubic phases is much less well understood. It is known that the fundamental properties of a bilayer can change upon addition of charged lipids ${ }^{48}$ and that lamellar phases incorporating charged lipids can swell indefinitely (although most systems reach an excess water point where the lattice parameter no longer increases and the mesophase coexists with bulk water).

A small number of previous studies have investigated the phase behaviour of MO when doped with a charged lipid, and with their swelling behaviour has been attributed to electrostatic interactions; ${ }^{24-27}$ however these investigations have been limited to temperatures below $20{ }^{\circ} \mathrm{C}$. The largest lattice parameter previously recorded is $268 \AA$ with approximately $2.5 \mathrm{~mol} \%$ distearoylphosphatidylglycerol (DSPG) in MO at 70 wt\% PIPES buffer. ${ }^{25}$

Fig. 3 and 4 show the phase behaviour and lattice parameter dependence of MO with $70 \mathrm{wt} \%$ water at 35 and $45{ }^{\circ} \mathrm{C}$, with increasing amount of DOPS or DOPG added to the system. Up until 5 mol\% charged lipid, both systems show very similar phase behaviour, adopting an $\operatorname{Im} 3 \mathrm{~m}$ phase which progressively swells with increasing concentrations of charged lipid. At $5 \mathrm{~mol} \%$ DOPS and DOPG, the systems reach a maximum lattice parameter of around $300 \AA$ and $350 \AA$ Respectively. At $10 \mathrm{~mol} \%$

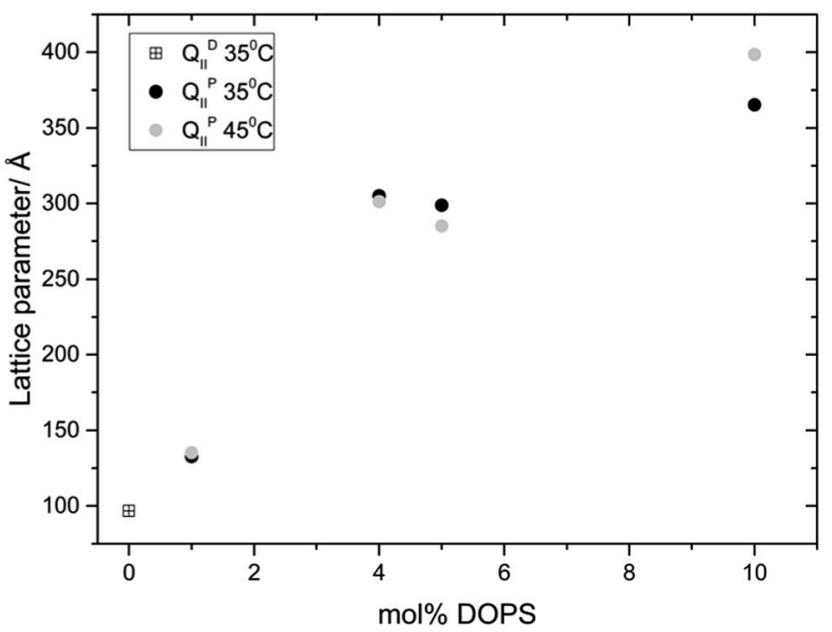

Fig. 3 Phase behavior and effect on the lattice parameter of incorporating DOPS into $\mathrm{MO}$.

charged lipid however the DOPG system collapses to a lamellar phase whilst the DOPS system swells further to reach a lattice parameter of $365 \AA$ at $35{ }^{\circ} \mathrm{C}$ and $399 \AA$ at $45{ }^{\circ} \mathrm{C}$.

In both systems, addition of just $1 \mathrm{~mol} \%$ charged lipid changes the bicontinuous cubic phase from $P n 3 m$ (as seen for pure MO) to $\operatorname{Im} 3 \mathrm{~m}$, which swells upon further addition of charged lipid. This phase transition is in agreement with previous work on doping monoacylglycerides with increasing amount of lipids, sterols or hydration enhancing agents. ${ }^{22,24-27}$ Increasing the concentration of charged lipid in the cubic structure will cause an increase in the surface charge density and so enhance the electrostatic repulsion between charged lipid headgroups. In addition, incorporating DOPS into the membrane may cause an increase in steric repulsion in the headgroup region due to its relatively large phosphatidylserine headgroup.

Both of these effects will cause the effective headgroup area to increase, resulting in a decrease in the magnitude of the

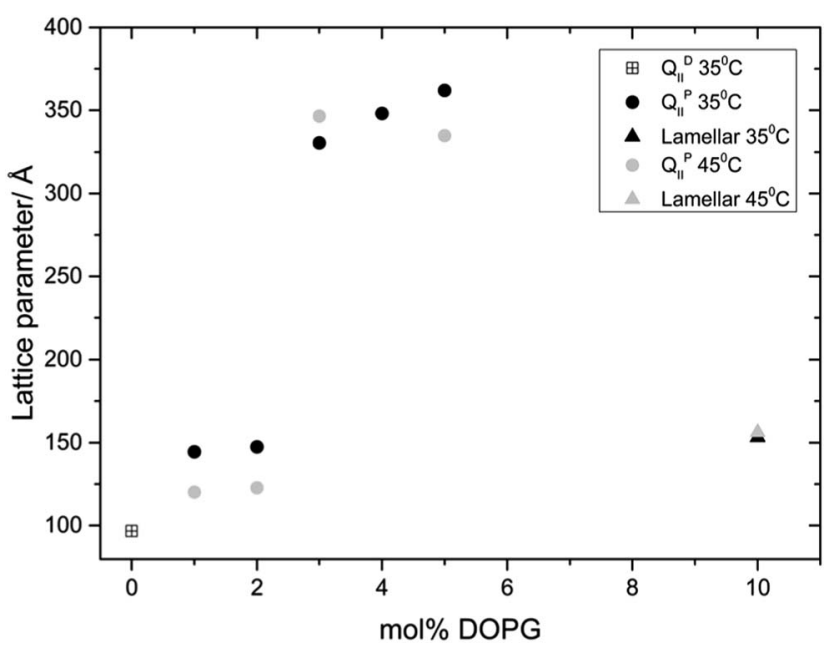

Fig. 4 Phase behavior and effect on the lattice parameter of incorporating DOPG into MO. 
monolayer spontaneous inverse curvature $\left(H_{0}\right)$ and hence a larger lattice parameter. Using eqn (1), it can be seen that increasing the headgroup area will decrease the packing parameter and decrease the magnitude of the Gaussian curvature $\langle K\rangle$ (i.e. becomes less negative), hence stabilizing the $\operatorname{Im} 3 m$ phase. ${ }^{43,44}$

The difference in phase behaviour between samples containing $10 \mathrm{~mol} \%$ DOPS (bicontinuous cubic $\operatorname{Im} 3 \mathrm{~m}$ phase) and DOPG (fluid lamellar phase) may be explained by considering the headgroup ordering of the charged lipids and their propensity to form hydrogen bonding interactions. NMR studies have shown that the molecular order, from ${ }^{2} \mathrm{H}$ NMR, in the headgroup region is significantly higher for DOPS than DOPG, which in turn dictates their propensity to form nonlamellar phases. ${ }^{48}$ As the DOPG headgroup is more disordered than DOPS it is likely to occupy a larger volume (with its hydration shell) resulting in a drive towards flatter structures. In addition, it is known that phosphatidylserine lipids can form hydrogen bonded networks ${ }^{49,50}$ resulting in a reduced effective headgroup size which drives increased inverse curvature (see ESI $\uparrow$ for further discussion of charged lipid spontaneous curvature).

Interestingly, dioleoylphosphatidic acid (DOPA) shows even greater headgroup ordering than DOPS but cannot form hydrogen bonded networks. Preliminary experiments incorporating $10 \mathrm{~mol} \%$ DOPA into MO show formation of an $\operatorname{Im} 3 \mathrm{~m}$ phase with a lattice parameter of $416 \AA$ at $45{ }^{\circ} \mathrm{C}$ which is larger than the equivalent DOPS structure. It appears that when doping MO with $10 \mathrm{~mol} \%$ charged lipid, DOPS forms the most curved structure (Im3m, $a=399 \AA$ A) followed by DOPA (Im3m, $a=416 \AA$ ) and the DOPG system forms a flat bilayer structure.

It should be noted that while the samples prepared here were hydrated with pure water, for some applications the use of buffers and salts are essential. In pure water, the only ions in solution are the charged lipid counterions $\left(\mathrm{Na}^{+}\right)$and so the electrostatic interactions between the charged lipids are maximised, however, increasing the ionic concentration of the aqueous solution by addition of salts or buffers will result in increased charge screening and a reduction in electrostatic interactions between the lipid bilayers. An increase in ionic concentration has been shown to cause a reduction in the lattice parameter observed in MO membranes with charged species incorporated..$^{26,27,51}$ In addition, it has been previously shown (and was observed here) that increasing the concentration of charged lipids in MO can causes a transition from a cubic to lamellar phase, ${ }^{24,27,52}$ however addition of buffers and salts causes a significant increase in the charged lipid concentration at which this transition occurs, due to a reduction in the inter-bilayer electrostatic interactions. For example, mixtures of MO with DOPG transform to a lamellar phase at 25\% DOPG in $10 \mathrm{mM}$ PIPES buffer and at 30\% DOPG when in $10 \mathrm{mM}$ PIPES and $1 \mathrm{M} \mathrm{NaCl},{ }^{53}$ however, in our pure water systems, $10 \%$ DOPG is sufficient to cause this transition to occur.

The effects of salt and buffer discussed above are likely to have a significant contribution from the effect of charge screening on the monolayer spontaneous curvature; it has been reported previously that in MO:DOPG and MO:DOPA mixtures, ${ }^{27,53}$ increasing electrostatic repulsion between the charged headgroups increase the effective lipid headgroup area resulting in a decrease of the magnitude of $H_{0}$ (see also additional discussion in the ESI $\dagger$ ). At low charged lipid concentration, this causes a reduction in the negative interfacial curvature of the cubic phase leading to an increase in the lattice parameter and above a critical charged lipid concentration will cause a transition to a lamellar phase. Increasing the ionic concentration will cause increased screening of the intra-monolayer electrostatic interaction leading to a reduction in cubic lattice parameters and an increase in the critical charged lipid concentration required to cause a phase transition to the lamellar phase.

Calculating the water volume fraction of these structures as described previously suggests that the maximum lattice parameter possible with $70 \mathrm{wt} \%$ water is $283 \AA$, which suggests that samples containing more than $2 \%$ charged lipid are not in excess water. This is not entirely consistent with the experimental data which show significant further swelling upon addition of charged lipid, however we have only used known parameters for MO for these calculations and it is very likely that volume and area parameters will change on incorporation of different lipids into MO and the model is known to become less accurate at water concentrations above $38 \mathrm{wt} \%{ }^{44}$

There is little temperature variation at low concentrations of charged lipid however at higher percentages the systems appear to swell with temperature, which is the opposite behaviour compared to pure MO. An increase in temperature will induce an increase in the chain splay (and hence an increase in $H_{0}$ ) resulting in a decrease in the lattice parameter of a mesophase. The opposite trend seen here suggests that electrostatic interactions are dominating the behaviour of the system. Studies have shown that DOPS membranes become thinner with an increase in temperature. ${ }^{54}$ Increased kinetic energy of the counterions as temperature is increased results in them dissociating and hence the screening length and the electrostatic repulsions in the headgroup also increase..$^{55}$ Electrostatic repulsions cause the headgroup area to increase, which in turn reduces $H_{0}$, leading to an increase in the lattice parameter. The calculated water channel diameters of the $\operatorname{Im} 3 \mathrm{~m}$ phase in the systems studied are presented in Fig. S1 (ESI $\dagger$ ). For the most swollen phase, the diameter of the water channels is calculated to be approximately $218 \AA$, which is almost three times larger than any swollen lipidic cubic phase recorded to date.

\section{Ternary mixtures of monoolein, cholesterol and charged lipid}

By doping MO with a small amount of charged lipid we can swell its cubic structure to 4 times larger than seen in pure MO via electrostatic repulsion across the water channels. Although thermal fluctuations will tend to destroy translational ordering for large lattice parameters, binary mixtures of $\mathrm{MO}$ with charged lipid somewhat resist this, due to long range electrostatic repulsions. To test whether thermal fluctuations are limiting the size of our cubic phases we added CHOL to the binary $\mathrm{MO}$ : charged lipid mixtures in order to stiffen the membrane whilst still keeping it fluid. By doing so we have managed to 
swell the cubic phase up to a maximum of approximately $470 \AA$ (around $70 \AA$ higher than the largest observed structure in corresponding binary mixtures containing DOPG or DOPS). A typical diffraction pattern of the $\operatorname{Im} 3 m$ phase obtained is shown in Fig. 5. Although by addition of cholesterol we have managed to reduce thermal fluctuations in the bilayer and further swell the structure, the rapid reduction in intensity of higher order diffraction peaks indicates that these fluctuations must still be significant. ${ }^{56}$

Fig. 6 shows the effect of increasing CHOL in ternary mixtures of MO while keeping the mol\% of charged lipid constant at $5 \mathrm{~mol} \%$. At $35{ }^{\circ} \mathrm{C}$ the DOPS mixture adopts an $\operatorname{Im} 3 \mathrm{~m}$ phase with a lattice parameter of $299 \AA$ at 0 mol\% CHOL and swells to a maximum of $455 \AA$ at $30 \mathrm{~mol} \% \mathrm{CHOL}$. At $45{ }^{\circ} \mathrm{C}$, the Im $3 m$ phase swells from $285 \AA$ at 0 mol\% CHOL to $467 \AA$ at $30 \mathrm{~mol} \% \mathrm{CHOL}$. At $30 \mathrm{~mol} \%$ the $\operatorname{Im} 3 \mathrm{~m}$ phase is always in coexistence with CHOL monohydrate crystals $(d=34 \AA)$ and at $45{ }^{\circ} \mathrm{C}$ it is also in coexistence with an $\mathrm{H}_{\text {II }}$ phase. At $35^{\circ} \mathrm{C}$ the DOPG mixture adopts an Im $3 m$ phase which swells from $361 \AA$ at $0 \mathrm{~mol} \% \mathrm{CHOL}$ to a maximum of $415 \AA$ at $15 \mathrm{~mol} \% \mathrm{CHOL}$. At $45{ }^{\circ} \mathrm{C}$, the $\operatorname{Im} 3 m$ phase swells from $364 \AA$ at $0 \mathrm{~mol} \% \mathrm{CHOL}$ to $423 \AA$ at $30 \mathrm{~mol} \% \mathrm{CHOL}$. At $30 \mathrm{~mol} \%$ the $\operatorname{Im} 3 \mathrm{~m}$ phase coexists with an $\mathrm{H}_{\mathrm{II}}$ phase and excess CHOL crystals at both temperatures.

It should be noted that mixtures with 15 and $20 \mathrm{~mol} \% \mathrm{CHOL}$ for $5 \mathrm{~mol} \%$ DOPS and 10-20 mol\% CHOL for $5 \mathrm{~mol} \%$ DOPG are often seen to coexist with an $\mathrm{H}_{\text {II }}$ phase, leading to a smaller lattice parameter as the phases compete for water. MO in excess water is known to hydrolyse (producing glycerol and oleic acid) resulting in the formation of an $\mathrm{H}_{\text {II }}$ phase. ${ }^{57}$ As the $\mathrm{H}_{\text {II }}$ phase is not always seen in mixtures with $10-20 \mathrm{~mol} \% \mathrm{CHOL}$, its formation in these samples may be due to small amounts of $\mathrm{MO}$ hydrolysis. In contrast, the $\mathrm{H}_{\mathrm{II}}$ phases observed in the corresponding $30 \mathrm{~mol} \%$ CHOL samples are always seen, these mixtures shows consistent phase behaviour which is reversible

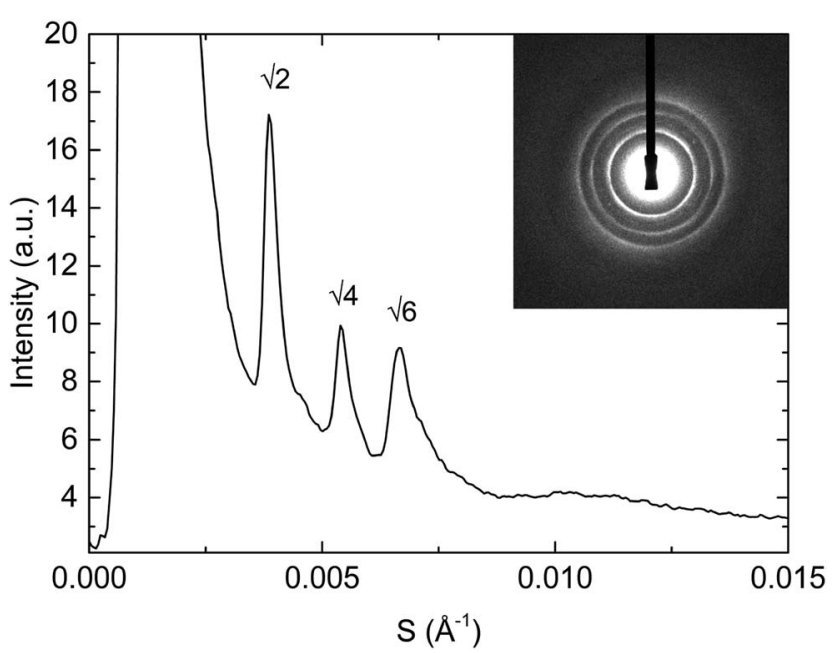

Fig. 5 Main figure: integrated synchrotron small angle $X$-ray diffraction pattern from the $1 \mathrm{~m} 3 \mathrm{~m}$ phase of hydrated $\mathrm{MO}$ : $\mathrm{CHOL}$ : DOPG $80: 15: 5$ mixture at $35{ }^{\circ} \mathrm{C}$ and indexing of the Bragg peaks. Inset: corresponding $2 \mathrm{D}$ diffraction pattern. Note that the scattering vector $S$ $=1 / d$.
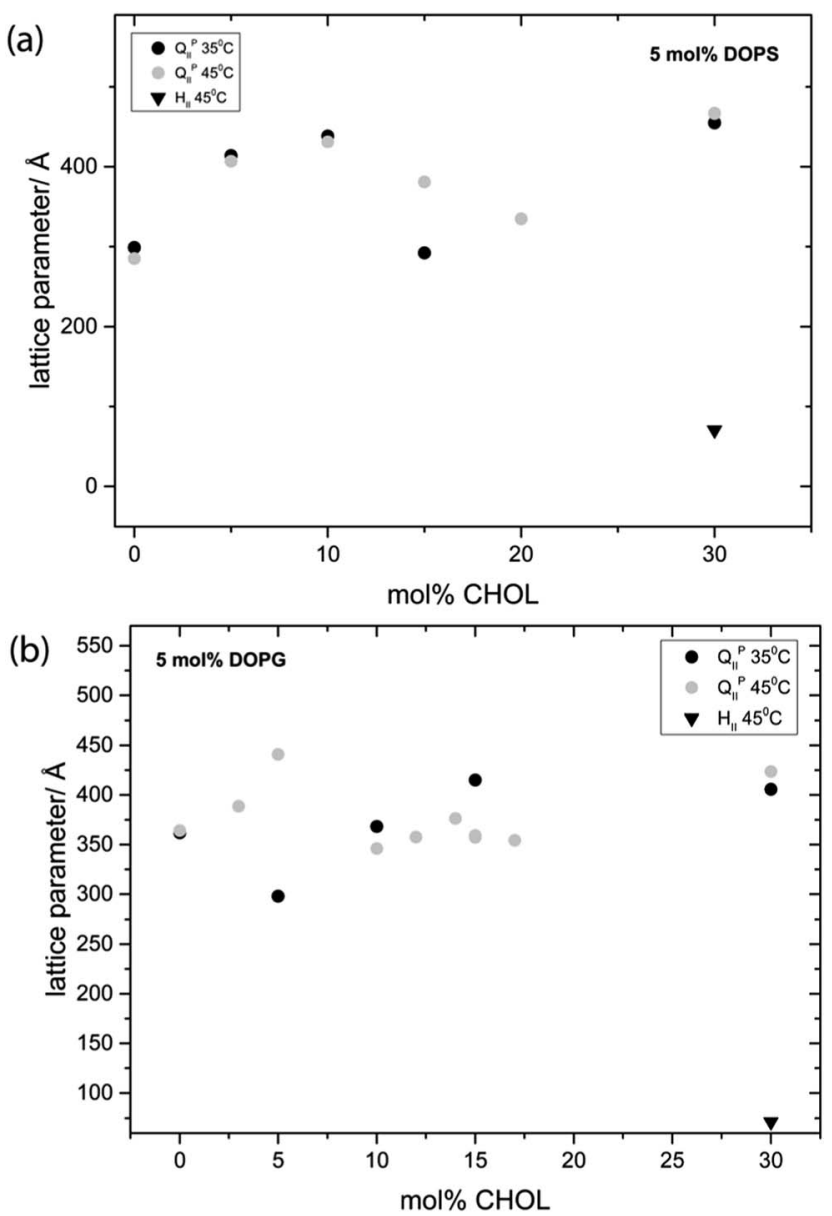

Fig. 6 Phase behavior and effect on the lattice parameter of incorporating $\mathrm{CHOL}$ into (a) MO : DOPS mixtures and (b) MO : DOPG mixtures, keeping the charged lipid concentration fixed at $5 \mathrm{~mol} \%$ in all samples.

when varying temperature and so we believe this is a true equilibrium structure. Interestingly, mixtures of MO with $5 \%$ $R-(+)$-limonene in excess water are also known to adopt an $\mathrm{H}_{\mathrm{II}}$ phase. ${ }^{58}$

Mixtures of MO with the concentration of DOPS fixed at $10 \mathrm{~mol} \%$ and varying amounts of $\mathrm{CHOL}$ have a relatively constant lattice parameter upon CHOL addition until the system transforms to a lamellar phase at $30 \mathrm{~mol} \% \mathrm{CHOL}$ (Fig. 7). All ternary mixtures containing $10 \mathrm{~mol} \%$ DOPG adopt a lamellar phase with lattice parameters in the region of $150 \AA$ (see Fig. S2, ESI $\dagger$ ). This behaviour is different to that seen with increasing $\mathrm{CHOL}$ at $5 \mathrm{~mol} \%$ DOPS. We believe that at $10 \mathrm{~mol} \%$ DOPS, the cubic phase swelling effect is dominated by the large electrostatic interactions, meaning that the membrane stiffening induced by CHOL incorporation has relatively little effect on the lattice parameter.

It should be noted that several of the ternary compositions were measured during independent synchrotron experiments and lattice parameters for these agree to within better than $30 \AA$. This variation is likely to be due to small differences in sample preparation. We believe the small fluctuations for $5 \mathrm{~mol} \%$ 


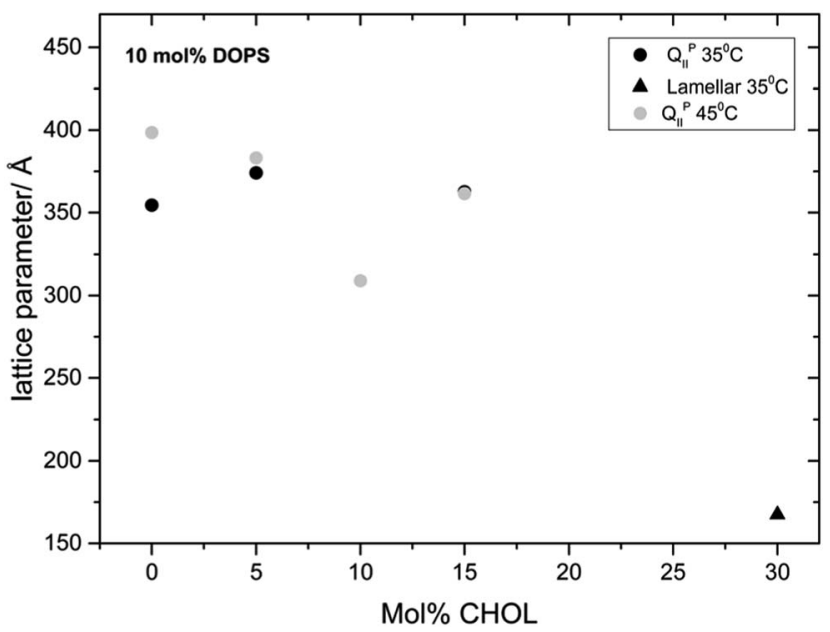

Fig. 7 Phase behavior and effect on the lattice parameter of incorporating $\mathrm{CHOL}$ into $\mathrm{MO}$ : DOPS mixtures keeping the concentration of DOPS fixed at $10 \mathrm{~mol} \%$.

DOPG and between 10 and $20 \mathrm{~mol} \% \mathrm{CHOL}$ at $45{ }^{\circ} \mathrm{C}$ are within this experimental variation.

As an example, the calculated water channel diameters of the Im $3 m$ phase for mixtures of MO with 5\% DOPS and varying CHOL concentration are presented in Fig. S3 (ESI $\dagger$ ). For the most swollen phase, the diameter of the water channels is calculated to be around $249 \AA$, which is the largest we have managed to achieve in any of the mixtures.

To the best of our knowledge, the lattice parameters and water channel diameters achieved here are the largest ever obtained for a bicontinuous cubic phase formed from a pure lipid mixture at equilibrium. The anionic lipids act synergistically with CHOL when doped into MO to swell the cubic phase significantly beyond structures obtained in binary mixtures of MO with either additional component.

\section{Experimental}

Detailed procedures can be found in the ESI. $\dagger$

Appropriate amounts of dry lipids were co-dissolved in chloroform. They were initially dried under a nitrogen stream then under vacuum for at least $24 \mathrm{~h}$. The samples were then hydrated with 70 wt\% HPLC grade water, subjected to a minimum of 20 freeze-thaw cycles (between -210 and $40{ }^{\circ} \mathrm{C}$ ) and at least 5 pressure cycles ( 1 to 2000 bar) homogenize the mixtures.

X-ray experiments were carried out on beamline ID02 at the European Synchrotron Radiation Facility (ESRF) and beamline I22 at Diamond Light Source (DLS) using X-ray wavelengths of $0.73 \AA$ (ESRF) or $0.69 \AA$ (DLS) and at camera lengths of $1.2 \mathrm{~m}$, $3.0 \mathrm{~m}, 4.0 \mathrm{~m}$ and $4.5 \mathrm{~m}$.

\section{Conclusions}

We have been able to generate and control swollen, protein free, bicontinuous cubic phases of spacegroup $\operatorname{Im} 3 m$ by (a) increasing the bilayer stiffness whilst still keeping it fluid by adding CHOL and (b) inducing electrostatic repulsion across the water channels by addition of anionic lipids to MO. By controlling the composition of the ternary mixtures we can tune the pore sizes of the structure and have managed to achieve lattice parameters of up to $470 \AA$ which is over 5 times larger than the cubic phase of MO in excess water. We hope these results will pave the way to developing engineering rules for the formation of bicontinuous cubic phases with lattice parameters significantly higher than those currently available. In addition, we have recently been able to dynamically tune the size of these structures using temperature and pressure changes. ${ }^{31}$ These highly swollen, tuneable cubic phases are likely to open up a wide range of bio-technical applications including formation of tuneable photonic crystals, high surface area catalysts, large molecule capture devices and scaffolds for in-cubo crystallization of large membrane proteins.

\section{Acknowledgements}

We thank ESRF and Diamond Light Source for the award of synchrotron beamtime and Dr Michael Sztucki (ESRF) and Dr Andy Smith (DLS) for their support and assistance. This work was supported by EPSRC Programme Grant EP/J017566/1, EPSRC Platform Grant EP/G00465X/1, by EPSRC Centre for Doctoral Training Studentships from the Institute of Chemical Biology EP/F500076/1 awarded to ESP and NLCM, and an EPSRC Doctoral Prize Fellowship awarded to AIIT.

\section{Notes and references}

1 Z. A. Almsherqi, S. D. Kohlwein and Y. Deng, J. Cell Biol., 2006, 173, 839-844.

2 G. K. Voeltz and W. A. Prinz, Nat. Rev. Mol. Cell Biol., 2007, 8, 258-264.

3 G. Basanez, F. M. Goni and A. Alonso, Biochemistry, 1998, 37, 3901-3908.

4 J. S. Patton and M. C. Carey, Science, 1979, 204, 145-148.

5 E. Staudegger, E. J. Prenner, M. Kriechbaum, G. Degovics, R. N. A. H. Lewis, R. N. McElhaney and K. Lohner, Biochim. Biophys. Acta, Biomembr., 2000, 1468, 213-230.

6 A. S. Andersson, L. Rilfors, G. Oradd and G. Lindblom, Biophys. J., 1998, 75, 2877-2887.

7 Y. R. Deng, M. Marko, K. F. Buttle, A. Leith, M. Mieczkowski and C. A. Mannella, J. Struct. Biol., 1999, 127, 231-239.

8 G. E. Schroder-Turk, S. Wickham, H. Averdunk, F. Brink, J. D. F. Gerald, L. Poladian, M. C. J. Large and S. T. Hyde, J. Struct. Biol., 2011, 174, 290-295.

9 F. Caboi, T. Nylander, V. Razumas, Z. Talaikyte, M. Monduzzi and K. Larsson, Langmuir, 1997, 13, 5476-5483.

10 I. Amar-Yuli, D. Libster, A. Aserin and N. Garti, Curr. Opin. Colloid Interface Sci., 2009, 14, 21-32.

11 C. Leal, N. F. Bouxsein, K. K. Ewert and C. R. Safinya, J. Am. Chem. Soc., 2010, 132, 16841-16847.

12 Y. Doi, A. Takai, Y. Sakamoto, O. Terasaki, Y. Yamauchi and K. Kuroda, Chem. Commun., 2010, 46, 6365-6367. 
13 A. Angelova, M. Ollivon, A. Campitelli and C. Bourgaux, Langmuir, 2003, 19, 6928-6935.

14 M. Caffrey and V. Cherezov, Nat. Protoc., 2009, 4, 706-731.

15 M. Caffrey, Biochem. Soc. Trans., 2011, 39, 725-732.

16 R. F. Service, Science, 2014, 343, 1094-1097.

17 C. V. Kulkarni, T. Y. Tang, A. M. Seddon, J. M. Seddon, O. Ces and R. H. Templer, Soft Matter, 2010, 6, 3191-3194.

18 J. Briggs, H. Chung and M. Caffrey, J. Phys. II, 1996, 6, 723751.

19 A. Zabara and R. Mezzenga, Soft Matter, 2013, 9, 1010-1014.

20 A. Zabara and R. Mezzenga, Soft Matter, 2012, 8, 6535-6541.

21 B. Angelov, A. Angelova, M. Ollivon, C. Bourgaux and A. Campitelli, J. Am. Chem. Soc., 2003, 125, 7188-7189.

22 R. Negrini and R. Mezzenga, Langmuir, 2012, 28, 1645516462.

23 W. Sun, J. J. Vallooran, A. Zabara and R. Mezzenga, Nanoscale, 2014, 6, 6853-6859.

24 V. Cherezov, J. Clogston, Y. Misquitta, W. Abdel-Gawad and M. Caffrey, Biophys. J., 2002, 83, 3393-3407.

25 J. Engblom, Y. Miezis, T. Nylander, V. Razumas and K. Larsson, Prog. Colloid Polym. Sci., 2001, 116, 9-15.

26 U. Aota-Nakano, S. J. Li and M. Yamazaki, Biochim. Biophys. Acta, Biomembr., 1999, 1461, 96-102.

27 S. J. Li, Y. Yamashita and M. Yamazaki, Biophys. J., 2001, 81, 983-993.

28 A. Yaghmur, P. Laggner, S. G. Zhang and M. Rappolt, PLoS One, 2007, 2, e479.

29 D. P. Siegel and B. G. Tenchov, Biophys. J., 2008, 94, 39873995.

30 C. E. Conn, O. Ces, X. Mulet, S. Finet, R. Winter, J. M. Seddon and R. H. Templer, Phys. Rev. Lett., 2006, 96, 108102.

31 H. M. G. Barriga, A. I. I. Tyler, N. L. C. McCarthy, E. S. Parsons, O. Ces, R. V. Law, J. M. Seddon and N. J. Brooks, Soft Matter, 2015, 11, 600-607.

32 R. Bruinsma, J. Phys. II, 1992, 2, 425-451.

33 G. C. Shearman, O. Ces and R. H. Templer, Soft Matter, 2010, 6, 256-262.

34 Y. La, C. Park, T. J. Shin, S. H. Joo, S. Kang and K. T. Kim, Nat. Chem., 2014, 6, 534-541.

35 M. Dubois, T. Zemb, L. Belloni, A. Delville, P. Levitz and R. Setton, J. Chem. Phys., 1992, 96, 2278-2286.

36 G. van Meer, D. R. Voelker and G. W. Feigenson, Nat. Rev. Mol. Cell Biol., 2008, 9, 112-124.

37 J. E. Smotrys and M. E. Linder, Annu. Rev. Biochem., 2004, 73, 559-587.
38 K. Simons and E. Ikonen, Nature, 1997, 387, 569-572.

39 A. M. Powl, J. M. East and A. G. Lee, Biochemistry, 2008, 47, 12175-12184.

40 C. R. Loomis, G. G. Shipley and D. M. Small, J. Lipid Res., 1979, 20, 525-535.

41 D. L. Gater, V. Reat, G. Czaplicki, O. Saurel, A. Milon, F. Jolibois and V. Cherezov, Langmuir, 2013, 29, 8031-8038.

42 P. O. Eriksson and G. Lindblom, Biophys. J., 1993, 64, 129136.

43 S. T. Hyde, J. Phys. Chem., 1989, 93, 1458-1464.

44 R. H. Templer, J. M. Seddon, P. M. Duesing, R. Winter and J. Erbes, J. Phys. Chem. B, 1998, 102, 7262-7271.

45 D. C. Turner, Z. G. Wang, S. M. Gruner, D. A. Mannock and R. N. Mcelhaney, J. Phys. II, 1992, 2, 2039-2063.

46 D. M. Anderson, S. M. Gruner and S. Leibler, Proc. Natl. Acad. Sci. U. S. A., 1988, 85, 5364-5368.

47 M. Pisani, S. Bernstorff, C. Ferrero and P. Mariani, J. Phys. Chem. B, 2001, 105, 3109-3119.

48 G. Lindblom, L. Rilfors, J. B. Hauksson, I. Brentel, M. Sjolund and B. Bergenstahl, Biochemistry, 1991, 30, 10938-10948.

49 H. I. Petrache, S. Tristram-Nagle, K. Gawrisch, D. Harries, V. A. Parsegian and J. F. Nagle, Biophys. J., 2004, 86, 15741586.

50 I. R. Miller and M. Eisenstein, Bioelectrochemistry, 2000, 52, 77-81.

51 S. M. Masum, S. J. Li, Y. Tamba, Y. Yamashita, T. Tanaka and M. Yamazaki, Langmuir, 2003, 19, 4745-4753.

52 M. Yamazaki, in Advances in Planar Lipid Bilayers and Liposomes, ed. A. L. Liu, Academic Press, 2009, vol. 9, pp. 163-209.

53 T. S. Awad, Y. Okamoto, S. M. Masum and M. Yamazaki, Langmuir, 2005, 21, 11556-11561.

54 P. Szekely, T. Dvir, R. Asor, R. Resh, A. Steiner, O. Szekely, A. Ginsburg, J. Mosenkis, V. Guralnick, Y. Dan, T. Wolf, C. Tamburu and U. Raviv, J. Phys. Chem. B, 2011, 115, 14501-14506.

55 D. Andelman, in Structure and dynamics of membranes, ed. R. Lipowsky and E. Sackmann, Elsevier Science, Amsterdam, New York, 1995, pp. 603-641.

56 D. Roux and C. R. Safinya, J. Phys., 1988, 49, 307-318.

57 F. Caboi, G. S. Amico, P. Pitzalis, M. Monduzzi, T. Nylander and K. Larsson, Chem. Phys. Lipids, 2001, 109, 47-62.

58 A. Salonen, S. Guillot and O. Glatter, Langmuir, 2007, 23, 9151-9154. 